IRA-International Journal of Technology \& Engineering

ISSN 2455-4480; Vol.03, Issue 03 (2016)

Institute of Research Advances

http://research-advances.org/index.php/IRAJTE

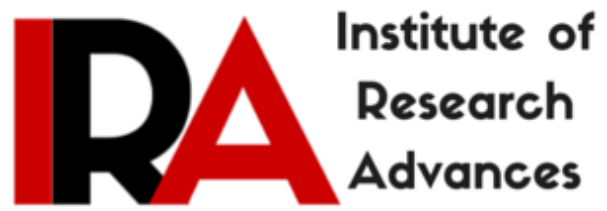

\title{
Performance of Combination of Texture and Object Based Techniques in Image Classification for Urban Land Cover
}

\section{Ms. J. Jacinth Jennifer}

PG Student: Department of Civil Engineering

Anna University Regional Campus

Tirunelveli, India.

DOI: http://dx.doi.org/10.21013/jte.v3.n3.p1

How to cite this paper:

Jennifer, J. (2016). Performance of Combination of Texture and Object Based

Techniques in Image Classification for Urban Land Cover. IRA-International Journal of

Technology \& Engineering (ISSN 2455-4480), 3(3).

doi:http://dx.doi.org/10.21013/jte.v3.n3.p1

(C) Institute of Research Advances

\section{(c) ) EY-NC}

This works is licensed under a Creative Commons Attribution-Non Commercial 4.0 International License subject to proper citation to the publication source of the work.

Disclaimer: The scholarly papers as reviewed and published by the Institute of Research Advances (IRA) are the views and opinions of their respective authors and are not the views or opinions of the IRA. The IRA disclaims of any harm or loss caused due to the published content to any party. 
Satellite imagery paves way to obtain tangible information through remote sensing techniques. It is necessary to classify the image in order to extract the features. There exist various classification techniques and algorithms to retrieve various features from imagery. As the technology development proceeds in a faster track it is necessary to compensate its advancements by developing new techniques for feature retrieval. As far as high resolution satellite imagery are concerned object based feature retrieval and texture based feature retrieval techniques are gaining its importance. The texture based feature retrieval has various techniques involved in it, among which Haralick's texture parameters has much importance. Thereby object based technique also has its own way of algorithms and processes for feature retrieval. The eCognition software provides a platform for combining texture and object based technique. It is well known from various journals that object based technique is best for classifying high resolution imagery. Thus the image is primarily segmented into objects for classification. The Haralick's texture parameters which serve well in classification of urban land cover is chosen by computing statistical analysis. Finally the chosen texture parameter is adopted in the classification of the objects. The classified imagery is checked for accuracy and a high accuracy of $94.5 \%$ is obtained.

Keywords: texture; object; satellite imagery; haralick's texture parameters; statistical analysis; urban feature extraction; classification

\section{INTRODUCTION}

The object based feature retrieval technique is gaining its importance as the resolution of the satellite imagery increases. Earlier in low resolution images a single pixel holds a specific feature whereas in high resolution imagery, many pixels together comprises a feature. Thus clubbing the pixels together as objects serve good in feature extraction. The segmentation process clusters the pixels into groups forming objects. Further processes are carried out over the objects so as to classify the imagery or extract a specified feature.

While describing texture based feature retrieval it is important to notify that, the texture parameters takes the spatial relationship (i.e.) the information about the spatial arrangement of intensities or colours into account. Thus both spatial and spectral characteristics are considered in texture based technique.

Thereby taking into study the performance of object based technique combined with texture based technique is expected to give better accuracy in image classification. The eCognition software paves a finest platform to exhibit and experiment the behavior of the combination of these techniques.

\section{DATA USED}

High resolution satellite imagery of an urban land cover is required for the study. The IKONOS imagery of SanDiego city, California, USA is used, which provides a spatial resolution of $1 \mathrm{~m}$. SanDiego is one among the important cities of California which has a dense urban land cover. Fig. 1 is the true colour IKONOS imagery of a part of SanDiego city. 


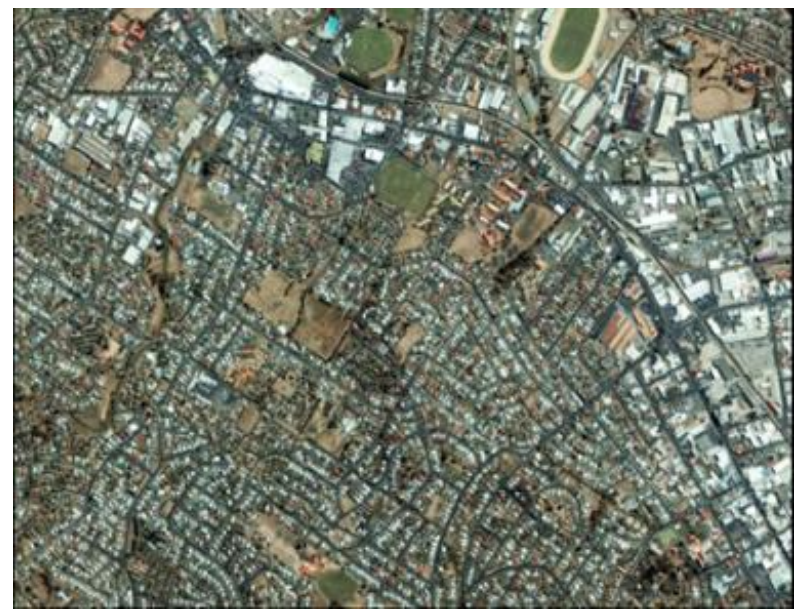

Figure 1. IKONOS image of SanDiego city.

\section{METHODOLOGY}

Primarily the optimum texture parameter has to be statistically analyzed and known. There are nearly thirteen Haralick's texture parameters. Each of the texture layer is computed and analyzed individually for various urban features. The statistical computations are made for each urban feature so as to examine the optimal texture parameter for urban feature retrieval. The statistical mean and range were chosen for statistical analysis.

In eCognition platform, the segmentation process is carried out so as to split the image into objects. Over the object layer, the classification is carried out based on the chosen texture parameter. Finally the classified image is checked for accuracy. Fig. 2 describes the methodology in a form of flow chart.

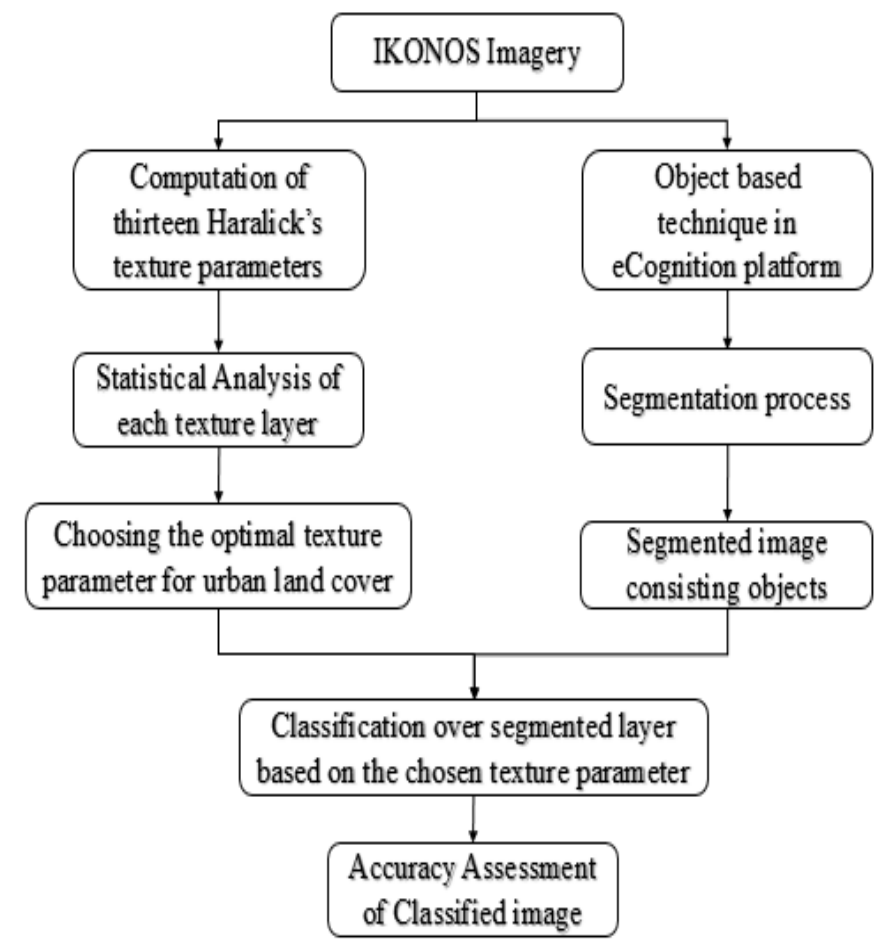

Figure 2. Methodology Flow Chart. 


\section{RESULTS AND DISCUSSION}

\section{A. Statistical Examination of Texture Parameters}

The texture analysis is performed over the IKONOS imagery so as to examine the capacity of each texture parameter in urban feature extraction. The statistical mean of each layer over various urban features proved essential in identifying the optimal texture parameter. The Haralick texture parameter, GLCM Mean is found to be of considerable importance because of its considerable variation in each direction from fig 3 .
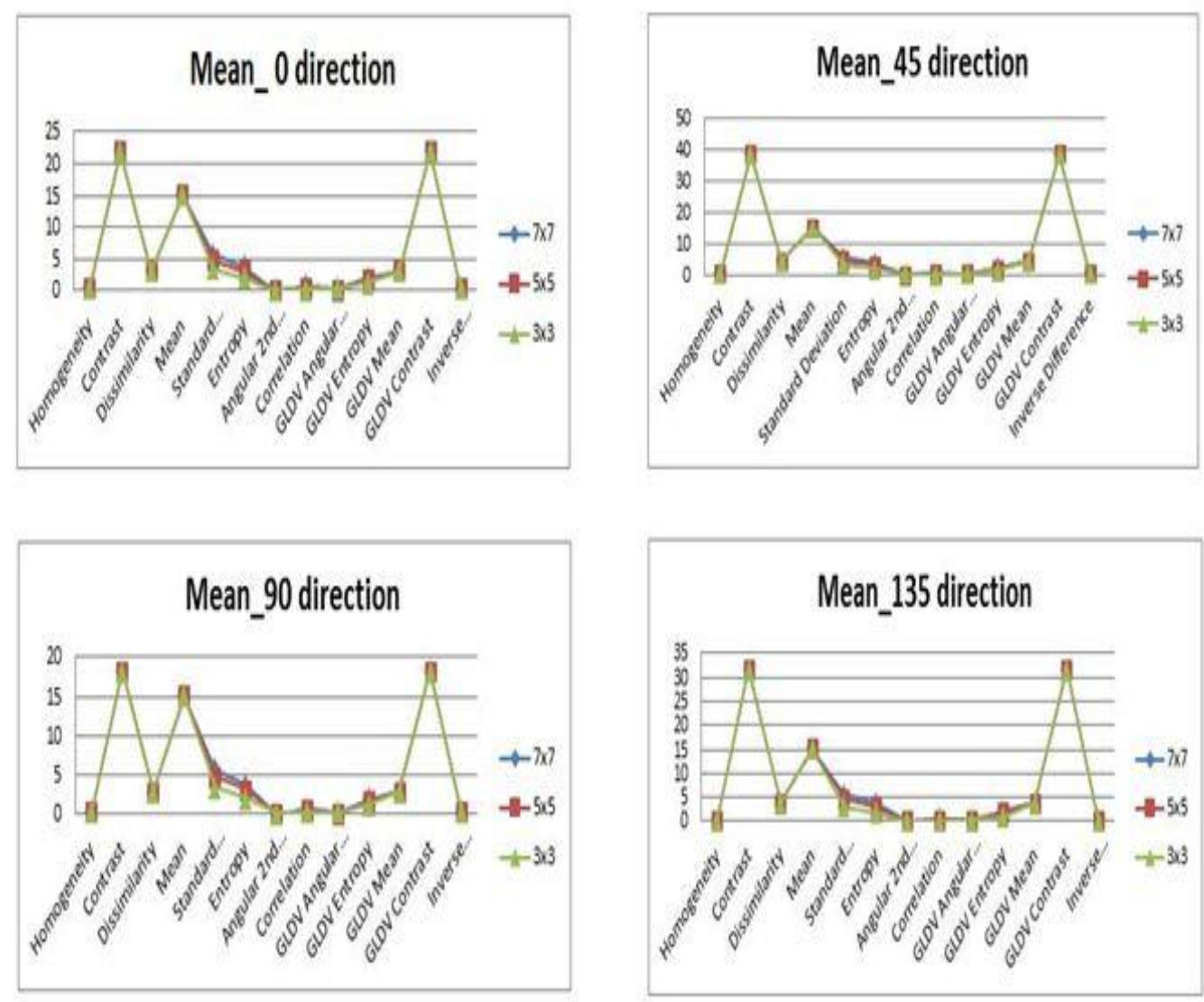

Figure 3. Results form statistical Analysis.

\section{B. Segmentation Process}

The preliminary step involving object based technique is segmentation. Multi resolution segmentation algorithm is used for segmentation. An optimal scale, shape factor and compactness are also prescribed in order to obtain optimal objects essential for classification of urban land cover.

\section{Classification and Accuracy Assessment}

The classification is thus carried out over the segmented imagery. Based on the GLCM Mean values, the urban features such as Road, Tree, Barren land, Concrete Building, Red Tiled building, Wooden building and Shadow were classified. Fig. 4 shows the classified image.

Finally in order to validate the performance of the technique involving both texture and object based technique, accuracy assessment is carried over the classified image and an accuracy of $94.5 \%$ is obtained. 


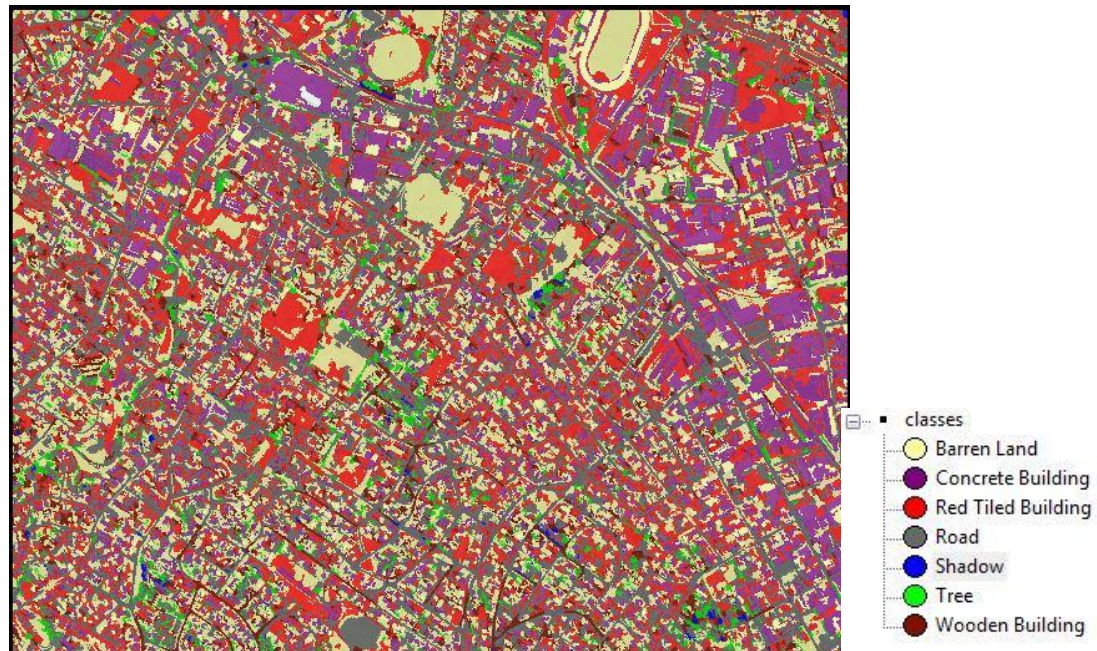

Figure 4. Classified image.

\section{CONCLUSION}

The urban land cover classification is done in an effective manner with a very good accuracy of $94.5 \%$ adopting the texture and object based technique together in a single platform. This efficiency proves the efficiency of the combination of two new techniques. Thus it is evident that high resolution satellite imageries has a better technique for image classification than the traditional ones.

\section{REFERENCES}

[1] Blaschke.T (2010), 'Object-Based Image Analysis for Remote Sensing', ISPRS Journal of Photogrammetry and Remote Sensing, Vol.65, pp.2-16.

[2] Jitendra Malik, Serge Belongie, Thomas Leung and Jianbo Shi (2001), "Contour and Texture Analysis for Image Segmentation", International Journal of Computer Vision, Vol.43, No.1, pp.7-27.

[3] Lucy Cheposgei Chepkochei (2011), 'Object-oriented Image Classification of Individual Trees using ERDAS Imagine Objective: Case Study of Wanjohi area, Lake Naivasha Basin, Kenya', Kenya Geothermal Conference 2011.

[4] Manuel A. Aguilar, Andrea Vallario, Fernando J. Aguilar, Andres Garcia Lorca and Claudio Parente (2015), 'Object-based Greenhouse Horticultural Crop Identification from Multi-temporal Satellite Imagery: A Case Study in Almeria, Spain', Remote Sensing, Vol.7, pp.7378-7401.

[5] Martin Herold, Joseph Scepan, Andre Muller and Sylvia Gunther (2003), 'Objectoriented Mapping and Analysis of Urban Land use/cover using IKONOS data', Geoinformation for European-wide Integration, Benes(ed.), pp.531-538.

[6] Paidamwoyo Mhangara and John Odindi (2013), 'Potential of Texture-based Classification in Urban Landscapes using Multispectral Aerial Photos', South African Journal of Science, Vol.109, No.3, pp.1-8.

[7] Robert M. Haralick, K.Shanmugam and ITS'HAK Dinstein (1973), “Textural Features for Image Classification", IEEE Transactions on Systems, Man and Cybernetics, Vol.3, No.6, pp.610-621.

[8] Shervan Fekri Ershad (2011), 'Colour Texture Classification Approach based on Combination of Primitive Pattern Units and Statistical Features', The International Journal of Multimedia and its Applications (IJMA), Vol.3, No.3. 
[9] Vishal S. Thakare, Nitin N. Patil and Jayshri S. Sonawane (2013), 'Survey on Image Texture Classification Techniques', International Journal of Advancements in Technology, Vol.4, No.1, pp.97-104.

[10] Yun Zhang (2001), 'Texture-Integrated Classification of Urban Treed Areas in High-Resolution Colour-Infrared Imagery', Photogrammetric Engineering and Remote Sensing, Vol.67, No.12, pp.1359-1365. 